\title{
A NEW MIXING RULE BASED ON A STATISTICAL MECHANICAL PERTURBATION THEORY APPLICABLE TO NONPOLAR ASYMMETRIC MIXTURES
}

\author{
YOSHIMORI MIYANO \\ Department of Applied Chemistry, Okayama University of Science, Okayama 700 \\ HIROKATSU MASUOKA \\ Department of Chemical Engineering, Hiroshima University, Higashihiroshima 724
}

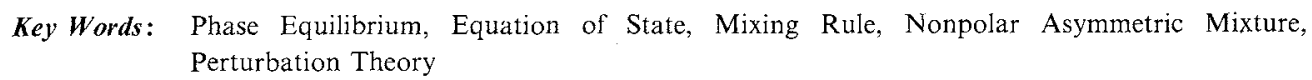

\begin{abstract}
A new mixing rule based on a statistical mechanical perturbation theory is proposed. It gives similar results to the van der Waals one-fluid model for mixtures of equisized molecules. A generalized equation of state utilizing the new mixing rule is applied to the calculation of high-pressure vapor-liquid equilibria for nonpolar asymmetric mixtures.

Comparisons are made with experimental data for binary systems containing hydrogen. The agreement of calculated and experimental high pressure vapor-liquid equilibria is rather good.
\end{abstract}

\section{Introduction}

Recently, much attention has been given to extending an equation of state (EOS) for pure fluids to mixtures. The general procedure for such extensions is to use the van der Waals one-fluid model ${ }^{28)}$ (vdW1). The vdW1 gives good results for symmetric systems.

For extension to asymmetric systems, a local composition, two-fluid model (LC) was studied by many authors. ${ }^{9.17,29,30)}$ It seems important how rigorously characteristics of the attractive interaction between molecules are evaluated. Usually the LC is empirically treated to obtain good results in industrial calculations.

A statistical mechanical perturbation theory gives reasonable results for mixtures of molecules that differ in size. In this paper, a new mixing rule based on a statistical mechanical perturbation theory is proposed to extend an EOS for pure fluids to mixtures of asymmetric molecules that differ extremely in size. The generalized EOS proposed previously ${ }^{16)}$ is used in the calculation of high-pressure vapor-liquid equilibria.

\section{A New Mixing Rule}

The Barker-Henderson perturbation theory ${ }^{11}$ gives reasonable results for Lennard-Jones potential fluids. The first-order Helmholtz free energy for mixtures is expressed as follows:

Received March 23, 1984. Correspondence concerning this article should be addressed to Y. Miyano.

$$
\frac{A_{1}^{M}}{N k T}=\frac{2 \pi \rho}{k T} \sum_{i} \sum_{j} x_{i} x_{j}\left[\int_{d_{i j}}^{\infty} u_{i j} g_{i j}^{h s} r^{2} d r-\int_{d_{i j}}^{\sigma_{i j}} u_{i j} g_{i j}^{h S} r^{2} d r\right]
$$

where $u_{i j}$ is the Lennard-Jones potential and $g_{i j}^{h s}$ is the radial distribution function of hard-sphere mixtures. The first term of Eq. (1) is rewritten as follows:

$$
\begin{aligned}
& \int_{d_{i j}}^{\infty} u_{i j} g_{i j}^{h s} r^{2} d r=4 \varepsilon_{i j} d_{i j}^{3} S_{i j} \\
& S_{i j}=I_{i j}^{(12)} / c_{i j}^{12}-I_{i j}^{(6)} / c_{i j}^{6} \\
& I_{i j}^{(n)}=\int_{1}^{\infty} \frac{1}{t_{i j}^{n-2}} g_{i j}^{h s} d t_{i j} \\
& c_{i j}=\frac{d_{i j}}{\sigma_{i j}}=\int_{0}^{1}\left[1-\exp \left(-\frac{u_{i j}^{*}}{T_{i j}^{*}}\right)\right] d\left(\frac{r}{\sigma_{i j}}\right)=f\left(T_{i j}^{*}\right)
\end{aligned}
$$

where $t_{i j}=r / d_{i j}, T_{i j}^{*}=k T / \varepsilon_{i j}, u_{i j}^{*}=u_{i j} / \varepsilon_{i j}$ and $d_{i j}$ is the hard-sphere diameter. Since numerical calculation of Eq. (4) is time-consuming, it may not be applicable to industrial calculations as suggested by Rogers and Prausnitz. ${ }^{23)}$

In this work, $I_{i j}$ defined in Eq. (4) was empirically approximated from the analogy of the radial distribution function at $r=d_{i j}$ for hard-sphere mixtures ${ }^{11}$ as follows (see Appendix):

$$
\begin{aligned}
& I_{i j}^{(n)}=I_{\text {pure }}^{(n)}+\delta_{i j}\left[I_{\text {pure }}^{(n)}-\frac{1}{(1-y)(n-3)}\right] \\
& \delta_{i j}=(1-\alpha y) \ln \left(\sqrt{d_{i} d_{j}} \eta_{2} / \eta_{3}\right)
\end{aligned}
$$




$$
\begin{aligned}
& I_{\mathrm{pure}}^{(n)}=\int_{1}^{\infty} \frac{1}{t^{n-2}} g_{\mathrm{pure}}^{h s} d t=\sum_{i} a_{n i} y^{i-1} \\
& y=\frac{\pi}{6} \rho \sum_{i} x_{i} d_{i}^{3} \\
& \eta_{m}=\sum_{i} x_{i} d_{i}^{m}
\end{aligned}
$$

In this paper, Eq. (6) is called a new mixing rule. $\alpha$ in Eq. (7) is 1.1, which was determined from fitting to the exact $S_{i j}$ calculated with the Percus-Yevic equa$\operatorname{tion}^{11)}$ for the radial distribution function of hardsphere mixtures. $x_{i}$ is the mole fraction of component $i$ and $a_{n i}$ in Eq. (8) was given in the previous paper. ${ }^{31 \text { ) }}$ As $S_{22}$ is more sensitive to the vapor-liquid equilibria for mixtures than $S_{11}$, where $d_{1}<d_{2}$, $\alpha$ was determined to fit the exact $S_{22}$.

Figure 1 shows comparisons of equimolar $S_{i j}$ for a binary mixture, $d_{2} / d_{1}=2$. In these calculations, $c_{i j}$ is set at 0.95 . The dashed lines express the mean-density approximation $^{15)}$ results, where the integrals for mixtures are approximated with the corresponding purefluid integrals, and $I_{11}=I_{22}=I_{12}=I_{\text {pure }}$. Figure 2 shows comparisons of $S_{i j}$ at $y=0.3$ for $d_{2} / d_{1}=2$. As shown in these figures, Eq. (6) gives relatively good results with average deviations of $1.7,0.8$ and $0.7 \%$ for $S_{11}, S_{12}$ and $S_{22}$, respectively, in the regions of $0.1<y<0.4, \quad 1.2<d_{2} / d_{1}<3 \quad$ and $0.1<x_{1}<0.9$. Equation (6) will be also valid for variable $c_{i j}$ and for other potentials like the 6-18 potential.

\section{Equation of State for Mixtures}

The Helmholtz free energy for pure fluids is expressed by using the generalized EOS proposed previously ${ }^{16)}$ as follows:

$$
\begin{aligned}
& \frac{A}{N k T}=\frac{A_{h s}}{N k T}+\frac{A_{1}}{N k T}+\frac{A_{2}}{N k T} \\
& \frac{A_{1}}{N k T}=6 \times 3^{3 / 2} y\left(\frac{\gamma}{T^{*}}\right)\left[Z(y, c)_{\mathrm{pure}}^{(18)} / c^{18}-Z(y, c)_{\mathrm{pure}}^{(6)} / c^{6}\right] \\
& Z(y, c)_{\mathrm{pure}}^{(n)}=I_{\mathrm{pure}}^{(n)} \\
& \quad-\left[\frac{G_{1}-G_{2}}{n-3}\left(1-c^{n-3}\right)+\frac{G_{2}}{n-4}\left(1-c^{n-4}\right)\right] \\
& G_{1}=(1-y / 2) /(1-y)^{3} \\
& G_{2}=\sum_{i=1}^{4} b_{i} y^{i} \\
& \frac{A_{2}}{N k T}=-40.5\left(\frac{\gamma}{T^{*}}\right)^{2} y F\left(T^{*}, y\right)_{\mathrm{pure}} \\
& F\left(T^{*}, y\right)_{\mathrm{pure}}=\sum_{i=1}^{6} E_{i}\left(T^{*}\right) y^{i-1} \\
& E_{i}\left(T^{*}\right)=E_{i 1}+E_{i 2} T^{*}+E_{i 3} / T^{*}+E_{i 4} / T^{* 2}+E_{i 5} / T^{* 5}
\end{aligned}
$$

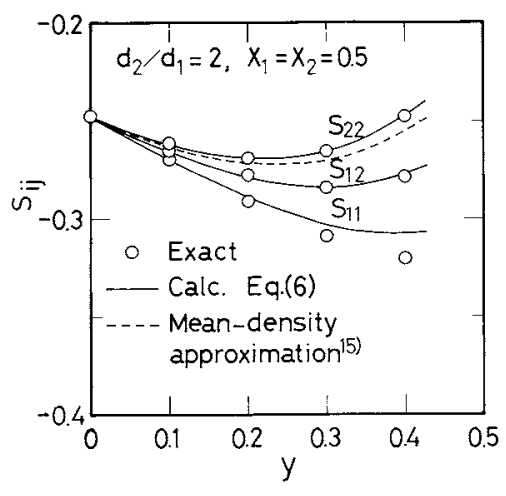

Fig. 1. Comparisons of calculated and exact $S_{i j}$ for equimolar model mixtures.

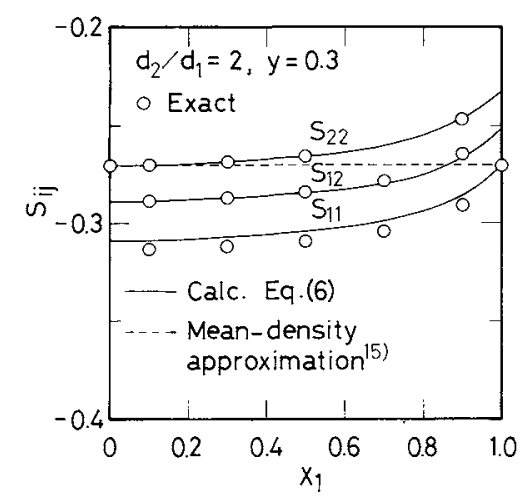

Fig. 2. Comparisons of calculated and exact $S_{i j}$ at $y=0.3$.

The values of $b_{i}$ and $E_{i j}$ were given in the previous paper. ${ }^{16)}$

The Helmholtz free energy for mixtures is generally expressed as follows:

$$
\frac{A^{M}}{N k T}=\frac{A_{h s}^{M}}{N k T}+\frac{A_{1}^{M}}{N k T}+\frac{A_{2}^{M}}{N k T}
$$

where $A_{h s}^{M}$ is the Helmholtz free energy of hard-sphere fiuids, the property of which was rigorously given by Mansoori et al. $^{14)}$ for the Carnahan-Starling EOS. ${ }^{3)}$ The Helmholtz free energy for perturbation terms is generally expressed as follows:

$$
\begin{aligned}
\frac{A_{1}^{M}}{N k T}= & 3^{3 / 2} \pi \rho \sum_{i} \sum_{j} x_{i} x_{j}\left(\frac{\gamma_{i j}}{T_{i j}^{*}}\right) d_{i j}^{3} \\
& \times\left(Z_{i j}^{(18) / c_{i j}^{18}}-Z_{i j}^{(6)} / c_{i j}^{6}\right) \\
\frac{A_{2}^{M}}{N k T}= & -\frac{27}{4} \pi \rho \sum_{i} \sum_{j} x_{i} x_{j}\left(\frac{\gamma_{i j}}{T_{i j}^{*}}\right)^{2} d_{i j}^{3} F_{i j}
\end{aligned}
$$

From the new mixing rule (Eq. (6)), $Z_{i j}^{(n)}$ and $F_{i j}$ are expressed as follows:

$$
\begin{aligned}
& Z_{i j}^{(n)}=Z\left(y, c_{M}\right)_{\mathrm{pure}}^{(n)}+\delta_{i j}\left[Z\left(y, c_{M}\right)_{\mathrm{pure}}^{(n)}-\frac{c_{M}^{n-3}}{(1-y)(n-3)}\right] \\
& F_{i j}=F\left(y, T_{M}^{*}\right)_{\mathrm{pure}}+\delta_{i j}\left[F\left(y, T_{M}^{*}\right)_{\mathrm{pure}}-\frac{E_{1}\left(T_{M}^{*}\right)}{(1-y)}\right]
\end{aligned}
$$




$$
\left.\begin{array}{l}
\varepsilon_{i j}=\left(1-k_{i j}\right) \sqrt{\varepsilon_{i} \varepsilon_{j}} ; \quad \sigma_{i j}=\left(\sigma_{i}+\sigma_{j}\right) / 2 \\
\gamma_{i j}=\sqrt{\gamma_{i} \gamma_{j}} ; \quad \gamma_{i}=\gamma_{0 i}+\gamma_{1 i} / T_{i}^{*}+\gamma_{2 i} / T_{i}^{* 2}
\end{array}\right\}
$$

The potential parameters for mixtures, which will be derived from the conformal-solution theory using the mean-density approximation, ${ }^{15)}$ were assumed to evaluate $\varepsilon_{M}$ in Eq. (25).

$$
\left.\begin{array}{c}
\varepsilon_{M} d_{M}^{3}=\sum \sum x_{i} x_{j} \varepsilon_{i j} d_{i j}^{3} \\
\gamma_{M} \varepsilon_{M} d_{M}^{3}=\sum \sum x_{i} x_{j} \gamma_{i j} \varepsilon_{i j} d_{i j}^{3} \\
\gamma_{M}^{2} \varepsilon_{M}^{2} d_{M}^{3}=\sum \sum x_{i} x_{j} \gamma_{i j}^{2} \varepsilon_{i j}^{2} d_{i j}^{3}
\end{array}\right\}
$$

In this paper, these types of equations are called parameter-mixing rules.

From thermodynamic considerations, the pressure is derived as follows:

$$
\begin{aligned}
& P=\rho^{2} k T[\partial(A / N k T) / \partial \rho]_{T, N} \\
& =P_{h s}+3^{3 / 2} \pi \rho^{2} k T \sum \sum x_{i} x_{j}\left(\frac{\gamma_{i j}}{T_{i j}^{*}}\right) d_{i j}^{3} \\
& \times\left(Z_{i j}^{(18)^{\prime}} / c_{i j}^{18}-Z_{i j}^{(6)^{\prime}} / c_{i j}^{6}\right) \\
& -\frac{27}{4} \pi \rho^{2} k T \sum \sum x_{i} x_{j}\left(\frac{\gamma_{i j}}{T_{i j}^{*}}\right)^{2} d_{i j}^{3} F_{i j}^{\prime}
\end{aligned}
$$

where

$$
\left.\begin{array}{l}
Z_{i j}^{(n) \prime}=\partial \rho Z_{i j}^{(n)} / \partial \rho \\
F_{i j}^{\prime}=\partial \rho F_{i j} / \partial \rho
\end{array}\right\}
$$

As pointed out by Mollerup, ${ }^{18)}$ mixing rules should reduce to a random-mixture model when fitted to random-mixture data. For mixtures of equisized molecules, $d_{i}=d_{j}, \delta_{i j}$ becomes zero and the free energy for the attractive part becomes as follows:

$$
\begin{aligned}
\frac{A_{1}^{M}}{N k T}= & 3^{3 / 2} \frac{\pi \rho}{k T} \sum \sum x_{i} x_{j} \gamma_{i j} \varepsilon_{i j} d_{i j}^{3}\left(Z\left(y, c_{M}\right)_{\text {pure }}^{(18)} / c_{i j}^{18}\right. \\
& \left.-Z\left(y, c_{M}\right)_{\mathrm{pure}}^{(6)} / c_{i j}^{6}\right) \\
\frac{A_{2}^{M}}{N k T}= & -\frac{27}{4} \frac{\pi \rho}{(k T)^{2}} F\left(y, T_{M}^{*}\right)_{\text {pure }} \sum \sum x_{i} x_{j}\left(\gamma_{i j} \varepsilon_{i j}\right)^{2} d_{i j}^{3}
\end{aligned}
$$

At low temperature limit, $c_{i j}$ approaches unity. Then Eq. (29) becomes:

$$
\begin{aligned}
\frac{A_{1}^{M}}{N k T}=3^{3 / 2} & \frac{\pi \rho}{k T}\left(Z\left(y, c_{M}\right)_{\text {pure }}^{(18)}-Z\left(y, c_{M}\right)_{\text {pure }}^{(6)}\right) \\
& \times \sum \sum x_{i} x_{j} \gamma_{i j} \varepsilon_{i j} \sigma_{i j}^{3}
\end{aligned}
$$

Equations (30) and (31) indicate that the new mixing rule gives similar expressions to the vdW1 model for mixtures of equisized molecules at low temperature limit.

$$
\gamma_{M} \varepsilon_{M} \sigma_{M}^{3}=\sum \sum x_{i} x_{j} \gamma_{i j} \varepsilon_{i j} \sigma_{i j}^{3}
$$

$$
\left(\gamma_{M} \varepsilon_{M}\right)^{2} \sigma_{M}^{3}=\sum \sum x_{i} x_{j}\left(\gamma_{i j} \varepsilon_{i j}\right)^{2} \sigma_{i j}^{3}
$$

and from the approximation in Eq. (26) and the definition of $y$, Eq. (9), the remaining parametermixing rules are:

$$
\left.\begin{array}{l}
\sigma_{M}^{3}=\sum x_{i} \sigma_{i}^{3}\left(=\sum \sum x_{i} x_{j} \sigma_{i j}^{3} \text { at } \sigma_{i}=\sigma_{j}\right) \\
\varepsilon_{M} \sigma_{M}^{3}=\sum \sum x_{i} x_{j} \varepsilon_{i j} \sigma_{i j}^{3}
\end{array}\right\}
$$

On the other hand, at high temperature limit, where $g_{i j}^{h s} \rightarrow 1$, the new mixing rule gives similar expressions to the vdW1 model for all kinds of nonpolar mixtures. It is expected at any temperature that the new mixing rule gives results for vapor-liquid equilibria for equisized mixtures similar to the results utilizing the vdW 1 type parameter-mixing rules.

At low densities, the EOS utilizing the proposed mixing rule gives the second virial coefficient as follows:

$$
\begin{aligned}
B_{M} / N_{a v}= & \frac{2}{3} \pi \sum \sum x_{i} x_{j} d_{i j}^{3}+3^{3 / 2} \pi \sum \sum x_{i} x_{j} \frac{\gamma_{i j}}{T_{i j}^{*}} d_{i j}^{3} \\
& \times\left(c_{M}^{15} / 15 c_{i j}^{18}-c_{M}^{3} / 3 c_{i j}^{6}\right) \\
& -\frac{27}{4} \pi \sum \sum x_{i} x_{j}\left(\frac{\gamma_{i j}}{T_{i j}^{*}}\right)^{2} d_{i j}^{3} E_{1}\left(T_{M}^{*}\right)
\end{aligned}
$$

Since $c_{M}$ and $T_{M}^{*}$ depend on mole fractions, Eq. (35) does not have a quadratic mole-fraction dependence. But it approximately equals the second virial coefficient having such dependence. The second virial coefficients calculated from Eq. (35) agreed well with those calculated from a quadratic mole-fraction dependent expression with an absolute average error of $1.9 \mathrm{~cm}^{3} / \mathrm{mol}$ for the $N_{2}-n$-heptane mixture, where the second virial coefficient for the mixture varies from -1300 to $25 \mathrm{~cm}^{3} / \mathrm{mol}$.

\section{Potential Parameters for Pure Substances}

The potential parameters for hydrogen, which is a quantum gas, were assumed as follows as suggested by Gunn et $a l^{8 \text { ) }}$

$$
\left.\begin{array}{c}
\varepsilon_{i j}=\varepsilon_{i j}^{c} /\left(1+21.8 / M_{i j} T\right) \\
\sigma_{i j}^{3}=\left(\sigma_{i j}^{c}\right)^{3} /\left(1-9.91 / M_{i j} T\right) \\
1 / M_{i j}=\left(1 / M_{i}+1 / M_{j}\right) / 2
\end{array}\right\}
$$

where $\varepsilon^{c}$ and $\sigma^{c}$ are the classical potential parameters. The parameters for hydrogen were determined from the vapor pressures ${ }^{21)}$ and PVT data $^{2)}$ by a method similar to that described previously. ${ }^{16)}$ The average deviations for vapor pressures ( 21 to $31.5 \mathrm{~K}$ ), PVT data (44 to $589 \mathrm{~K}, 0.1$ to $69 \mathrm{MPa}$ ) and saturated liquid densities $^{6)}(15$ to $27 \mathrm{~K}$ ) are $0.1,0.5$ and $4.2 \%$, respectively. The absolute error for the second virial coefficients $^{4)}$ is $7.7 \mathrm{~cm}^{3} / \mathrm{mol}$ at temperatures from 14 to $400 \mathrm{~K}$. The potential parameters for other sub- 
Table 1. Potential parameters for pure substances

\begin{tabular}{lcrrrr}
\hline Substance & $\varepsilon / k[\mathrm{~K}]$ & $\sigma \times 10^{10}[\mathrm{~m}]$ & \multicolumn{1}{c}{$\gamma_{0}$} & \multicolumn{1}{c}{$\gamma_{1}$} & \multicolumn{1}{c}{$\gamma_{2}$} \\
\hline $\mathrm{H}_{2}{ }^{*}$ & 39.492 & 2.8447 & 1.2009 & -0.0570 & 0.0699 \\
$\mathrm{~N}_{2}$ & 145.13 & 3.4801 & 0.8996 & 0.0099 & 0.0042 \\
$\mathrm{CO}_{2}$ & 409.45 & 3.5561 & 0.6628 & 0.0939 & -0.0007 \\
Methane & 210.04 & 3.6128 & 0.9590 & -0.0068 & 0.0044 \\
$n$-Hexane & 710.36 & 5.6809 & 0.5929 & 0.1237 & -0.0031 \\
$n$-Heptane & 767.35 & 5.9311 & 0.5461 & 0.1525 & -0.0057 \\
$n$-Decane & 897.06 & 6.7001 & 0.4358 & 0.2269 & -0.0124 \\
Benzene & 750.40 & 5.0089 & 0.6765 & 0.0883 & -0.0002 \\
Toluene & 810.63 & 5.3788 & 0.6308 & 0.1081 & -0.0018 \\
$m$-Xylene & 869.97 & 5.6944 & 0.5635 & 0.1436 & -0.0050 \\
\hline
\end{tabular}

\# Values of $\varepsilon$ and $\sigma$ for hydrogen are $\varepsilon^{c}$ and $\sigma^{c}$, respectively.

stances were obtained from the generalized correlations $^{16)}$ and are listed in Table 1.

\section{Calculation of High-Pressure Vapor-Liquid Equi- libria}

The generalized EOS for mixtures utilizing the proposed mixing rule, Eq. (6), is applied to the calculation of high-pressure vapor-liquid equilibria. In these calculations, the binary interaction parameters, $k_{i j}$, which are introduced in Eq. (24), were fitted to experimental liquid-phase compositions at experimental temperatures and pressures, and are listed in Table 2 for several systems. In phase equilibrium calculations, the chemical potential for component $i$ was obtained by numerical differentiations of free energy with respect to $N_{i}$.

$$
\mu_{i}=\left(\partial A / \partial N_{i}\right)_{T, N_{j \neq i}}
$$

Although an analytical expression for chemical potentials can be obtained from Eq. (37), it will be more troublesome. Figures 3 to $\mathbf{6}$ show typical results for calculated vapor-liquid equilibria. In Figs. 3 and 4, the calculated results for nonpolar mixtures which differ in size are compared with experimental data. ${ }^{5.13)}$ The solid lines express the calculated results using the new mixing rule, and one-point dashed lines express the calculated results with optimum interaction parameter using the vdWl type parameter-mixing rules for whole perturbed terms in Eqs. (11) to (18), which are expressed as follows:

$$
\left.\begin{array}{c}
\sigma_{M}^{3}=\sum \sum x_{i} x_{j} \sigma_{i j}^{3} \\
\varepsilon_{M} \sigma_{M}^{3}=\sum \sum x_{i} x_{j} \varepsilon_{i j} \sigma_{i j}^{3} \\
\gamma_{M} \varepsilon_{M} \sigma_{M}^{3}=\sum \sum x_{i} x_{j} \gamma_{i j} \varepsilon_{i j} \sigma_{i j}^{3}
\end{array}\right\}
$$

Agreement between experimental data and calculated results utilizing the new mixing rule is rather good.

In Figs. 5 and 6, the results for mixtures containing hydrogen are compared with experimental data. For the hydrogen (1)-methane (2) system, ${ }^{27)}$ the molecular size is appreciably different. At low tempera-

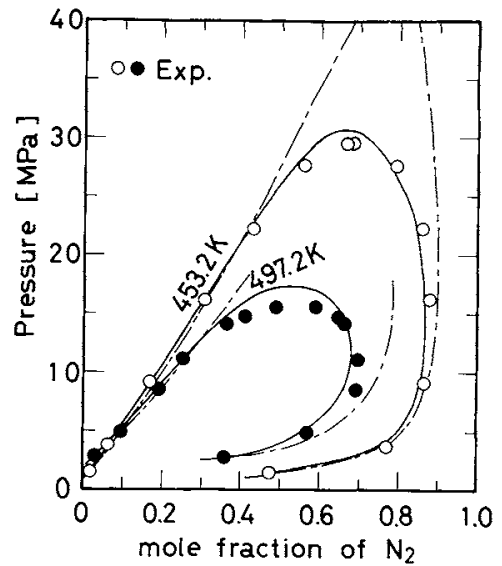

Fig. 3. Vapor-liquid equilibria for the $\mathrm{N}_{2}-n$-heptane system. ${ }^{5}$

Solid line, proposed mixing rule with optimum $k_{12}$; one-point dashed line, vdWl model with optimum $k_{12}$.

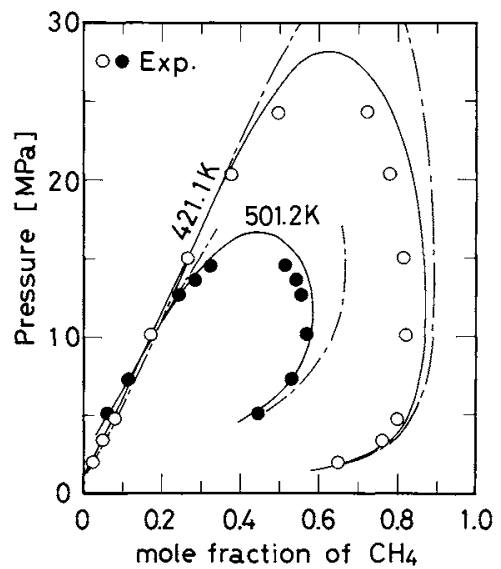

Fig. 4. Vapor-liquid equilibria for methane-benzene system. ${ }^{13}$ Solid and one-point dashed lines as in Fig. 3.

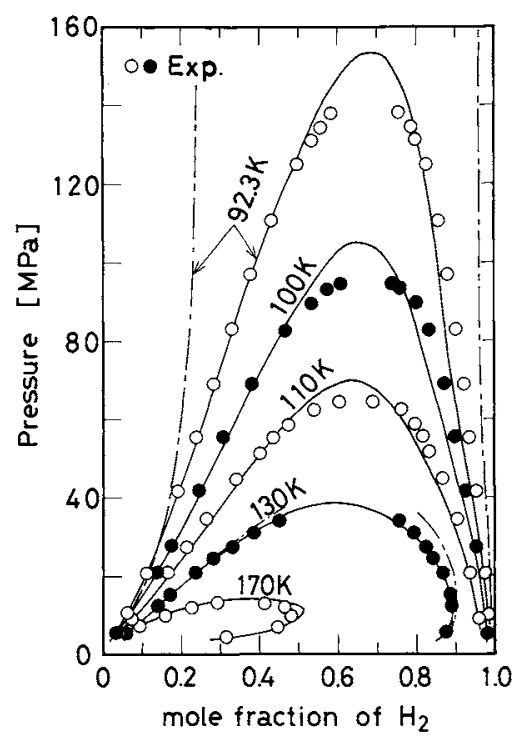

Fig. 5. Vapor-liquid equilibria for $\mathrm{H}_{2}-$-methane system. ${ }^{27)}$ Solid and one-point dashed lines as in Fig. 3.

tures, quantum effects may not be negligible. For the hydrogen (1)-n-heptane (2) system, ${ }^{22)}$ the ratios 
Table 2. Interaction parameters for several nonpolar mixtures

\begin{tabular}{|c|c|c|c|c|c|c|}
\hline System & $\begin{array}{c}\text { Temperature } \\
{[\mathrm{K}]}\end{array}$ & $\begin{array}{l}\text { Maximum } \\
\text { pressure } \\
{[\mathrm{MPa}]}\end{array}$ & $\begin{array}{l}k_{12} \\
{[-]}\end{array}$ & $\Delta x^{*} \times 100$ & $\Delta y^{* *} \times 100$ & $N D^{* * * *}$ \\
\hline \multirow[t]{6}{*}{$\mathrm{H}_{2}-$ Methane $^{271}$} & 92.3 & 130. & -0.005 & 0.78 & 1.94 & 10 \\
\hline & 100.0 & 90. & -0.009 & 1.41 & 1.98 & 9 \\
\hline & 110.0 & 62. & -0.014 & 1.72 & 2.17 & 8 \\
\hline & 130.0 & 34. & -0.021 & 0.88 & 0.75 & 9 \\
\hline & 150.0 & 22. & -0.03 & 1.08 & 2.82 & 7 \\
\hline & 170.0 & 12. & -0.04 & 0.52 & 3.02 & 5 \\
\hline \multirow{3}{*}{$\mathrm{H}_{2}-n$-Heptane 22$)$} & 424.15 & 80. & 0.185 & 0.36 & 0.40 & 8 \\
\hline & 471.65 & 70. & 0.12 & 0.75 & 1.83 & 10 \\
\hline & 498.85 & 35. & 0.08 & 0.56 & 3.29 & 8 \\
\hline \multirow[t]{4}{*}{$\mathrm{H}_{2}-n$-Decane ${ }^{24\}}$} & 462.45 & 25 . & 0.28 & 0.17 & 0.36 & 7 \\
\hline & 503.35 & 25. & 0.26 & 0.24 & 0.73 & 7 \\
\hline & 542.95 & 25 . & 0.24 & 0.26 & 2.03 & 7 \\
\hline & 583.45 & 25 . & 0.20 & 1.94 & 1.76 & 5 \\
\hline \multirow{2}{*}{$\mathrm{H}_{2}-$ Toluene $^{10\}}$} & 542.15 & 32. & 0.08 & 0.36 & 1.49 & 6 \\
\hline & 568.15 & 28 & 0.05 & 0.60 & 2.82 & 4 \\
\hline $\mathrm{N}_{2}-n$-Heptane ${ }^{5}$ & $453.15-497.15$ & 27. & 0.14 & 1.47 & 2.10 & 18 \\
\hline \multirow[t]{2}{*}{$\mathrm{CO}_{2}-n$-Hexane ${ }^{12 t}$} & $313.15-353.15$ & 11. & 0.19 & 0.79 & 0.29 & 19 \\
\hline & 393.15 & 12. & 0.18 & 0.33 & 0.61 & 8 \\
\hline \multirow[t]{3}{*}{$\mathrm{CO}_{2}-n$-Decane $e^{25)}$} & 462.55 & 5. & 0.245 & 0.14 & 0.16 & 4 \\
\hline & 542.95 & 5. & 0.23 & 0.07 & 1.07 & 4 \\
\hline & 583.65 & 5. & 0.215 & 0.23 & 1.50 & 4 \\
\hline $\mathrm{CO}_{2}-\mathrm{Toluene}^{26\}}$ & $393.25-542.85$ & 5. & 0.145 & 0.28 & 2.55 & 12 \\
\hline $\mathrm{CO}_{2}-m-\mathrm{Xylene}^{19\}}$ & $394.3-477.6$ & 17. & 0.155 & 1.15 & 0.63 & 16 \\
\hline \multirow[t]{2}{*}{ Methane $n$-Decane ${ }^{13)}$} & 542.75 & 13. & 0.14 & 0.30 & 1.35 & 4 \\
\hline & 583.05 & 9. & 0.12 & 1.13 & 2.87 & 5 \\
\hline Methane-Benzene $^{131}$ & $421.05-501.15$ & 24. & 0.07 & 1.10 & 3.81 & 18 \\
\hline Methane-Toluene ${ }^{13)}$ & $422.45-543.15$ & 25. & 0.085 & 1.10 & 2.08 & 25 \\
\hline Methane-m-Xylene ${ }^{191}$ & $394.3-477.6$ & 14. & 0.09 & 1.09 & 0.71 & 21 \\
\hline \multicolumn{7}{|c|}{$* \quad \Delta x=\left[\sum_{i=1}^{N D}\left(x_{\mathrm{ca} 1}-x_{\mathrm{exp}}\right)^{2} / N D\right]^{1 / 2} ; x$ is mole fraction of solute in liquid phase } \\
\hline \multicolumn{7}{|c|}{ ** $\quad \Delta y=\left[\sum_{i=1}^{N D}\left(y_{\text {cal }}-y_{\text {exp }}\right)^{2} / N D\right]^{1 / 2} ; y$ is mole fraction of solute in vapor phase } \\
\hline *** ND: Number of data $\mathrm{p}$ & oints & & & & & \\
\hline
\end{tabular}

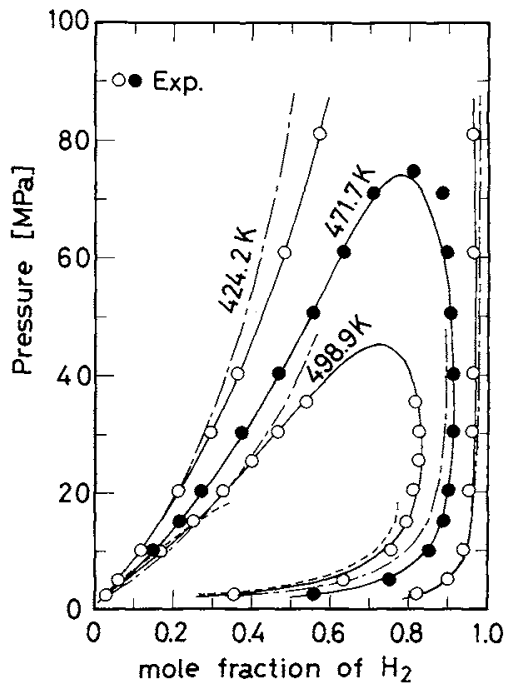

Fig. 6. Vapor-liquid equilibria for $\mathrm{H}_{2}-n$-heptane system. ${ }^{22}$ ) Solid and one-point dashed lines as in Fig. 3. Dashed lines express results using GT method. $\sigma_{2} / \sigma_{1}$ and $\varepsilon_{2} / \varepsilon_{1}$ are 2.1 and 19.4 , respectively. Although quantum effects may be negligible at high temperatures, the molecules differ extremely in size and well depth of potential function for this system.

The generalized EOS utilizing the vdW1 type parameter-mixing rules using temperature-dependent parameters for quantum gases gives poor results for such asymmetric systems. On the other hand, same EOS utilizing the new mixing rule was successfully applied to correlate high pressure vapor-liquid equilibria.

The interaction parameters for nonpolar asymmetric mixtures, especially for systems containing hydrogen, depended on temperature as listed in Table 2 , and smooth relations between interaction parameters and temperatures were obtained as suggested by Nishiumi. ${ }^{20)}$ This is probably due to empirical treatment of quantum effects, inaccuracy of EOS and uncertainties in mixing rules.

For other nonpolar asymmetric systems listed in Table 2, the calculated vapor-liquid equilibria utiliz- 


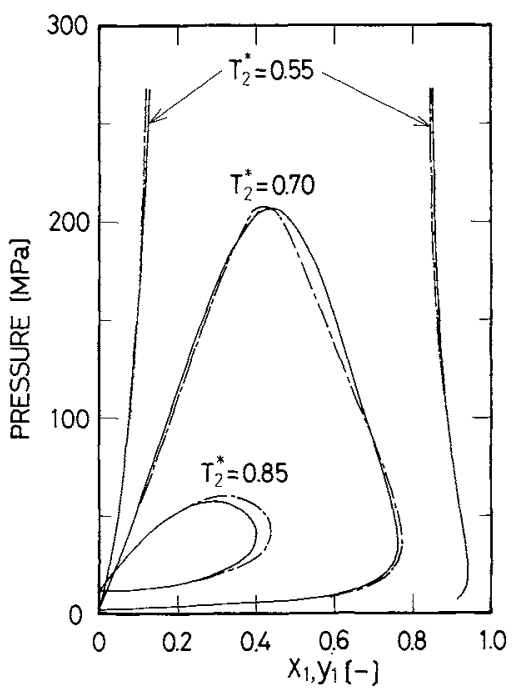

Fig. 7. Vapor-liquid equilibria for equisized model mixtures; $d_{2} / d_{1}=1\left(\sigma_{1}=3.6 \times 10^{-10} \mathrm{~m}\right), \varepsilon_{2} / \varepsilon_{1}=4\left(\varepsilon_{1} / k=200 \mathrm{~K}\right)$, $\gamma_{2}=\gamma_{1}=1$. Lines as in Fig. 3 .

ing the new mixing rule agreed with experimental data, as well as for the systems discussed above. In this table, comparisons of calculated and experimental vapor and liquid compositions at experimental temperatures and pressures except near the critical points for mixture are made. The third column in Table 2 shows the maximum pressures for which comparisons were made.

Another method to extend an EOS for pure fluids to mixtures, proposed by Gubbins and $\mathrm{Twu}^{7)}(\mathrm{GT})$, is also tested, where the mean-density approximation was used. Utilizing this approximation, $Z_{i j}$ and $F_{i j}$ are expressed as follows:

$$
\left.\begin{array}{c}
Z_{i j}^{(n)}=Z\left(y_{M}, c_{i j}\right)_{\text {pure }}^{(n)} \\
F_{i j}=F\left(y_{M}, T_{i j}^{*}\right)_{\text {pure }}
\end{array}\right\}
$$

Equation (39) was applied to calculations of vaporliquid equilibria for the $\mathrm{H}_{2}-n$-heptane system at 498.9 K. As shown by dashed lines in Fig. 6, this method gives poor results for mixtures which differ extremely in size, and fails to converge at high pressures.

In Fig. 7, the calculated results utilizing the new mixing rule are compared with the results utilizing parameter-mixing rules, Eq. (38), for equisized model mixtures. The results utilizing the new mixing rule deviate slightly from the results utilizing parametermixing rules, Eq. (38). Although Eqs. (32), (33) and (34) should all be used in the determination of potential parameters for mixtures, it is impossible to satisfy all equations with the three parameters $\varepsilon_{M}$, $\sigma_{M}$ and $\gamma_{M}$. This is probably the reason why the two results deviate slightly from each other.

\section{Conclusion}

The generalized equation of state proposed previously has been applied successfully to the calculation of high-pressure vapor-liquid equilibria for nonpolar asymmetric mixtures containing hydrogen, utilizing a new mixing rule (Eq. (6)) based on statistical mechanical perturbation theory. For mixtures of equisized molecules, this mixing rule gives results similar to those of the vdW1 model.

This mixing rule may be empirically applicable to other perturbed type equations like the van der Waals equation of state.

\section{Appendix}

The rigorous expression of the radial distribution function at $r=d_{i j}$ for hard-sphere mixtures ${ }^{11)}$ is:

$$
g_{i j}^{h s}\left(d_{i j}\right)=g_{\mathrm{pure}}^{h s}(d)+\left(\frac{2 d_{i} d_{j}}{d_{i}+d_{j}} \frac{\eta_{2}}{\eta_{3}}-1\right)\left[g_{\mathrm{pure}}^{h s}(d)-\frac{1}{1-y}\right]
$$

We assume $g_{i j}^{h s}(r)$ as follows:

$$
g_{i j}^{h s}(r)=g_{\text {pure }}^{h s}(r)+\delta_{i j}\left[g_{\text {pure }}^{h s}(r)-\frac{1}{1-y}\right]
$$

Eq. (A-2) may be a good approximation in the range of $r \simeq d$. By

\begin{tabular}{|c|c|c|}
\hline \multicolumn{3}{|c|}{ Nomenclature } \\
\hline$A$ & $=$ Helmholtz free energy & {$[\mathrm{J}]$} \\
\hline$a_{n i}$ & $=$ constant, Eq. (8) & {$[-]$} \\
\hline$B$ & $=$ second virial coefficient & {$\left[\mathrm{m}^{3} / \mathrm{mol}\right]$} \\
\hline$b_{i}$ & $=$ constant, Eq. (15) & {$[-]$} \\
\hline$c$ & $=$ cut-off parameter & {$[-]$} \\
\hline$d$ & $=$ effective hard-sphere diameter & {$[\mathrm{m}]$} \\
\hline$E$ & $=$ constant, Eq. (18) & {$[-]$} \\
\hline$g$ & $=$ radial distribution function & {$[-]$} \\
\hline$k$ & $=$ Boltzmann constant & {$[\mathrm{J} / \mathrm{K}]$} \\
\hline$M$ & $=$ relative molecular mass & {$[-]$} \\
\hline$N$ & $=$ number of molecules & {$[-]$} \\
\hline$N_{a v}$ & $=$ Avogadro constant & {$[1 / \mathrm{mol}]$} \\
\hline$P$ & $=$ pressure & {$[\mathrm{Pa}]$} \\
\hline$r$ & $=$ intermolecular distance & {$[\mathrm{m}]$} \\
\hline$T$ & $=$ temperature & {$[\mathrm{K}]$} \\
\hline$t$ & $=r / d$ & {$[-]$} \\
\hline$u$ & $=$ intermolecular potential & {$[\mathrm{J}]$} \\
\hline$x$ & $=$ mole fraction & {$[-]$} \\
\hline$y$ & $=$ reduced density & {$[-]$} \\
\hline$y$ & $=$ mole fraction in vapor phase, Fig. 7 & {$[-]$} \\
\hline$\alpha$ & $=$ parameter defined in Eq. (7) & {$[-]$} \\
\hline$\gamma$ & $=$ third parameter & {$[-]$} \\
\hline$\delta$ & $=$ parameter defined in Eq. (7) & {$[-]$} \\
\hline$\varepsilon$ & $=$ well depth in potential function & {$[\mathrm{J}]$} \\
\hline$\eta$ & $=$ parameter defined in Eq. (10) & {$[-]$} \\
\hline$\mu$ & $=$ chemical potential & {$[\mathrm{I}]$} \\
\hline$\rho$ & $=$ number density & {$\left[\mathrm{l} / \mathrm{m}^{3}\right]$} \\
\hline$\sigma$ & $=$ collision diameter & {$[\mathrm{m}]$} \\
\hline
\end{tabular}
inserting Eq. (A-2) into Eq. (4), Eq. (6) is obtained, where the form of $\delta_{i j}$ is empirically determined from fitting to the exact $S_{i j}$, which is expressed by Eq. (7). 


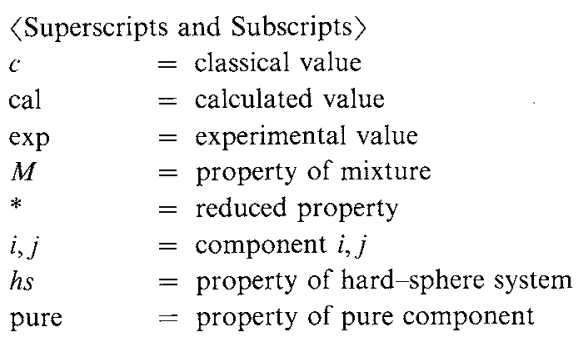

\section{Literature Cited}

1) Barker, J. A. and D. Henderson: J. Chem. Phys., 47, 4714 (1967).

2) Canjar, L. N. and F. S. Manning: "Thermodynamic Properties and Reduced Correlations for Gases," Gulf Pub. Co., Texas (1967).

3) Carnahan, N. F. and K. E. Starling: J. Chem. Phys., 51, 635 (1969).

4) Dymond, J. H. and E. B. Smith: "The Virial Coefficients of Gases," Clarendon Press, Oxford (1969).

5) Figuiere, P., J. F. Hom, S. Laugier, H. Renon, D. Richon and H. Szwarc: $A I C h E J ., 26,872$ (1980).

6) Francis, A. W.: Chem. Eng. Sci., 10, 37 (1959).

7) Gubbins, K. E. and C. H. Twu: Chem. Eng. Sci., 33, 863 (1978).

8) Gunn, R. D., P. L. Chueh and J. M. Prausnitz: AIChE J., 12, 937 (1966).

9) Huron, M. J. and J. Vidal: Fluid Phase Equilibria, 3, 255 (1979).

10) Laugier, S., D. Richon and H. Renon: J. Chem. Eng. Data, 25, 274 (1980).

11) Lebowitz, J. L.: Phys. Rev., 133, A895 (1964).

12) Li, Y. H., K. H. Dillard and R. L. Robinson, Jr.: J. Chem.
Eng. Data, 26, 53 (1981).

13) Lin, H. M., H. M. Sebastian, J. J. Simnick and K. C. Chao: J. Chem. Eng. Data, 24, 146 (1979).

14) Mansoori, G. A., N. F. Carnahan, K. E. Starling and T. W. Leland: J. Chem. Phys, 54, 1523 (1971).

15) Mansoori, G. A. and T. W. Leland: J. Chem. Soc., Faraday Trans. II, 68, 320 (1972).

16) Miyano, Y.: J. Chem. Eng. Japan, 16, 85 (1983).

17) Mollerup, J.: Fluid Phase Equilibria, 7, 121 (1981).

18) Mollerup, J.: Fluid Phase Equilibria, 15, 189 (1983).

19) Ng, H. J., S. S. S. Huang and D. B. Robinson; J. Chem. Eng. Data, 27, 119 (1982).

20) Nishiumi, H.: J. Chem. Eng. Japan, 16, 449 (1983).

21) Ohe, S.: "Computer Aided Data Book of Vapor Pressure," Data Book Pub. Co., Tokyo (1976).

22) Peter, S. and K. Reinhartz: Z. Phys. Chem. Frankfurt, 24, 103 (1960).

23) Rogers, B. L. and J. M. Prausnitz: Trans. Faraday Soc., 67, 3474 (1971)

24) Sebastian, H. M., J. J. Simnick, H. M. Lin and K. C. Chao: J. Chem. Eng. Data, 25, 68 (1980).

25) Sebastian, H. M., J. J. Simnick, H. M. Lin and K. C. Chao: J. Chem. Eng. Data, 25, 138 (1980).

26) Sebastian, H. M., J. J. Simnick, H. M. Lin and K. C. Chao: J. Chem. Eng. Data, 25, 246 (1980).

27) Tsang, C. Y., P. Clancy, J. C. G. Calado and W. B. Streett: Chem. Eng. Commun., 6, 365 (1980).

28) van der Waals, J. D.: Z. Phys. Chem., 5, 133 (1890).

29) Whiting, W. B. and J. M. Prausnitz: Fluid Phase Equilibria, 9, 119 (1982)

30) Won, K. W.: Fluid Phase Equilibria, 10, 191 (1983).

31) Yorizane, M. and Y. Miyano: J. Chem. Eng. Japan, 13, 433 (1980). 